(T1DM) and even precipitate a more severe presentation of DKA. It is also dangerous to fast a child with suspected diabetes insipidus. Reasons for GP referrals for FBTs were reviewed.

Methods A retrospective audit was conducted in 2015, assessing all GP referrals to the paediatric outpatient department (POPD) for FBTs from April to December. Subsequently, the POPD staff advised parents that FBTs were not required when they booked their blood tests. A re-audit was undertaken in 2016 from April to December.

Results 88 children were referred for FBTs in 2015, with a $42 \%$ reduction to 37 referrals in 2016. Ages ranged from 17 months to 16 years. Referrals were made from 36 GP surgeries (2015) and 26 GP surgeries (2016). In 2016, referrals were made from 31 different doctors. In each cycle, 1 patient had hypoglycaemia. The rest of the results were in the normal range (table 1).

\begin{tabular}{|c|c|c|}
\hline \multirow[t]{2}{*}{ Reason for FBT } & \multicolumn{2}{|c|}{ Number referred } \\
\hline & $2015(n=88)$ & $2016(n=37)$ \\
\hline ?T1DM & $7(8 \%)$ & $4(11 \%)$ \\
\hline Hypoglycaemia/hypoadrenalism & $1(1 \%)$ & $2(5 \%)$ \\
\hline Polyuria/polydipsia & $21(24 \%)$ & $10(27 \%)$ \\
\hline Lethargy & $11(13 \%)$ & $6(16 \%)$ \\
\hline Weight loss & $6(7 \%)$ & $1(3 \%)$ \\
\hline Recurrent infections & $10(11 \%)$ & $4(11 \%)$ \\
\hline Glycosuria & $1(1 \%)$ & $0(0 \%)$ \\
\hline
\end{tabular}

Conclusion Children were unnecessarily fasted: there were no positive results, however, there were two adverse outcomes of hypoglycaemia. Additionally, those with suspected T1DM were at risk of developing DKA.

Recommendations

- If suspecting T1DM, children should be referred to a paediatric team on the same day.

- A random blood glucose can be used for a diagnosis of T1DM.

- FBTs should not be undertaken for symptoms of hypoglycaemia.

- FBTs should not be undertaken in primary care settings as they can be dangerous.

- Regular updates/awareness (P)s for GPs regarding investigation of suspected T1DM and clinical risks of fasting in children.

- POPD staff should advise parents that FBTs are not required when they book in for blood tests.

- Re-audit.

\section{G223 HIGHER PERCENTAGE OF INSULIN PUMP USERS ACHIEVABLE BY A SMALL TEAM! EXPERIENCE OF PAEDIATRIC DIABETES TEAM AT ISLE OF MAN (IOM)}

A Gangadharan, P Unsworth, V Varshney, A Kurien. Paediatrics, Noble's Hospital, Douglas, Isle of Man, UK

10.1136/archdischild-2018-rcpch.218
Introduction Continuous subcutaneous Insulin infusion (CSII) using pump has revolutionised the management of diabetes in children. Studies have demonstrated that better metabolic control could be achieved using insulin pump. NICE recommends use of insulin pump in children with poor metabolic control, disabling hypoglycaemia or clinical situations where MDI use is not practical.

National Paediatric Diabetes Audit (NPDA) reports serves as yardstick to compare individual unit performance against wider national data. The IOM, not part of NPDA cohort aims to provide highest quality of care by critically analysing its performances against national audit outcomes.

Aims

- To assess the prevalence of pump users and mean HbA1c on the Island

- Compare unit performance with NPDA report

- Identify the potential ways of promoting wider insulin pump use in T1D

Methodology Data of all diabetic patients from April 2015 March 2016 managed at Nobles hospital, IOM. Key outcomes such as age, percentage of pumps users, mean HbA1c, and prevalence of associated disorders was collected. All results were compared to NPDA report for the year 2015-2016.

Results [Combined England and Wales data in bold]

Total of 48 patients with T1D $[\mathrm{M}=25(52 \%)]$ with a median age of 13.2 years (range 6.2-19.0) at the final point of data collection. The mean HbA1c was $63.3 \mathrm{mmol} / \mathrm{mol}$ [68.3], $<58 \mathrm{mmol} / \mathrm{mol}$ was $31.2 \%$ [26.6\%] and $>80 \mathrm{mmol} / \mathrm{mol}$ was $6.2 \%$ [17.9\%]. A total of 90\% (43/48, 27-Animas, 15Omnipod and 1-Medtronic who moved from UK) were on pump compared to $\mathbf{2 8 \%}$ in England and Wales for the same period. Rest of them were on MDI. Associated coeliac disease (1) and thyroid disorder (1) was noted. There were 3 admissions of hyperglycaemia/ketosis during this period.

Conclusions

- Better glycaemic control is achieved in a smaller unit in spite of a very high percentage of pump users.

- Provision of only 2 different pumps helped in better utilisation of expertise and at the same time providing crucial choice for the users (tube Vs tubeless).

Though part funding from charity organisation helps in procuring the pumps, it does not guarantee long term sustainability and hence need for liberal funding options by NHS.

\section{G224 DEVELOPMENT OF CARDIOVASCULAR RISK FACTORS IN CHILDREN WITH TYPE 1 DIABETES - A LONGITUDINAL COHORT STUDY}

1,2S Jones, ${ }^{1,3} \mathrm{~A}$ Kanolkar, ${ }^{2} \mathrm{~K}$ Matyka, 4,5E Gevers, ${ }^{4} \mathrm{~J}$ Allgrove, ${ }^{1} \mathrm{~T}$ Stephenson, ${ }^{1} \mathrm{R}$ Amin. ${ }^{1}$ Great Ormond Street Institute of Child Health, University College London, London, UK; ${ }^{2}$ Warwick Medical School, University of Warwick, Coventry, UK; ${ }^{3}$ Institute of Environmental Medicine, Karolinska Institutet, Stockholm, Sweden; ${ }^{4}$ Department of Paediatric Endocrinology and Diabetes, Royal London Children's Hospital, Barts Health NHS Trust, London, UK; ${ }^{5}$ Centre for Endocrinology, Queen Mary University, London, UK

\subsection{6/archdischild-2018-rcpch.219}

Aims Cardiovascular disease is the leading cause of death in adult diabetes. Atherosclerosis is related to modifiable risk factors beginning in childhood but can be reversed. Screening for complications is recommended from 12 years in the UK. 
This study investigated the prevalence and evolvement from diagnosis of cardiovascular risk factors (hypertension, obesity (BMI), dyslipidaemia, albuminuria) in an ethnically diverse population of children with Type 1 diabetes (T1D).

Methods The study included all children diagnosed with T1D attending three paediatric diabetes clinics in East London between 2005-2015. Clinical and demographic information was collected prospectively during routine check-ups. Linear longitudinal mixed effects modelling (growth curve analyses) were used to analyse the development of cardiovascular risk factors from diagnosis. Models were adjusted for age, gender, ethnicity, diabetes clinic, glycated haemoglobin $\left(\mathrm{HbA} 1_{\mathrm{c}}\right)$.

Results Of 565 children $60 \%$ were non-white, of whom 8\% were Bangladeshi and $8 \%$ from Somali backgrounds. Mean age at diagnosis was 8.5 years (0.9-19.4). Mean length of follow-up was 4.3 years $(0-10.8)$. There was variation between clinics in commencing screening, range $8.8-13.8$ years $(\mathrm{p}<0.001)$.

$33 \%$ of all measures demonstrated an unhealthy BMI, 15\% revealed microalbuminuria, 66\% were above lipid targets with $5 \%$ reaching treatment thresholds. The frequency of abnormalities before or after 12 years of age was unchanged.

Longitudinal modelling revealed mean increases in BMI $(0.6$ $\mathrm{kg} / \mathrm{m}^{2}, 95 \% \mathrm{CI}: 0.6$ to 0.7$)$, blood pressure $(1.8 \mathrm{mmHg}, 1.5-$ $2.1)$, total cholesterol $(0.05 \mathrm{mmol} / \mathrm{mol}, 0.03-0.06)$ and low density lipoprotein (LDL) $(0.2 \mathrm{mmol} / \mathrm{mol}, 0.01-0.04)$ per year from diagnosis for the entire cohort. $\mathrm{HbA} 1_{c}$ directly affected all variables other than albuminuria. Results from subgroup analysis in children over 12 years old were similar but not statistically significant.

There was significant ethnic variation: Annual increments were greater for Bangladeshi children's cholesterol and LDL $(0.38 \mathrm{mmol} / \mathrm{mol}, \quad 0.1-0.6$ and $0.32 \mathrm{mmol} / \mathrm{mol}, \quad 0.09-0.55$ respectively) and Somali children's BMI $\left(1.6 \mathrm{~kg} / \mathrm{m}^{2}, 0.7-2.5\right)$ compared to White.

Conclusions Levels of abnormalities were above regional and national averages in children of all ages. Somali and Bangladeshi children had less favourable profiles, which supports findings of increased cardiovascular risk in these ethnicities. Commencing screening in younger children and introducing tailored interventions for ethnic minorities with T1D may help reduce cardiovascular events in adulthood.

\section{G225(P) DISORDERS OF SEXUAL DIFFERENTIATION CLINIC, OXFORD, UK}

${ }^{1} \mathrm{P}$ Ahmed, ${ }^{2} \mathrm{R}$ Romero, ${ }^{3} \mathrm{~F}$ Ryan. ${ }^{1}$ Paediatrics, Barnet General Hospital, London, UK; 2Paediatric Surgery, John Raddliffe Hospital, Oxford, UK; ${ }^{3}$ Paediatric Endocrinology, John Radcliffe Hospital, Oxford, UK

\subsection{6/archdischild-2018-rcpch.220}

Aim Disorders of sexual differentiation (DSD) are a heterogeneous group of genetic disorders with a mismatch of chromosomal, gonadal and phenotypic sex. The John Radcliffe Hospital is one of the largest and few DSD centres in the country, providing a highly specialised multi-disciplinary team service. The aims were to analyse the patient cohort with DSD under the care of the hospital, including their diagnoses, medical and surgical interventions and ascertain the strengths of the DSD service provided, and how the service can be improved to meet UK guidance on a DSD clinic.

Methods A retrospective review was conducted from 20122016 to identify the diagnoses and medical and surgical interventions of the DSD patients. The DSD clinic service was compared with UK guidance.

Results 46 patients were identified with 28 males, 18 females and $91 \%$ of patients having attended the DSD MDT clinic at least once. The average age of patients was 7.5 years. The number of patients with a genetic diagnosis was $96 \%$ and of those with a confirmed diagnosis, the most common diagnoses were partial androgen insensitivity syndrome (27\%) and congenital adrenal hyperplasia (25\%). The majority of patients were diagnosed by ambiguous genitalia- $85 \%$. Endocrinological problems were identified in $43 \%$ of patients, requiring input in the form of hormone replacement. Surgical intervention was required in $89 \%$ of patients, with hypospadias repair being the most common procedure. Currently, the DSD clinic involves input from surgery/urology, endocrinology and psychiatry, in order to devise a clinical management plan, as per UK guidance. Further areas for improvement include an endocrinologist to act as 'clinical lead' for newborns, improved links to adult services and an annual MDT meeting to review and discuss the patients and review the DSD clinic performance.

Conclusions The DSD MDT clinic meets many of the UK requirements. Importantly, an MDT clinic allows families to retrieve all results in one day with liaison between specialties for holistic care, and minimises time taken off work and school thus improving attendance, whilst forming a support group in the waiting room. Therefore, despite being a costly service, it is certainly worth continuing.

\section{G226(P) COELIAC DISEASE SURVEILLANCE IN CHILDREN AND YOUNG PERSONS WITH TYPE 1 DIABETES-ARE WE COMPLIANT?}

S Shah, J Smyth, V Simpson. Paediatrics, Southern Health and Social Care Trust, Portadown, UK

\subsection{6/archdischild-2018-rcpch.221}

Aims To evaluate current practice with regards screening for coeliac disease (CD) in children and young people with type 1 diabetes (T1D) within our Trust and to compare it with the other 6 hospitals within in the region. This also includes ongoing surveillance practice for $\mathrm{CD}$ at subsequent follow-ups until the young person is transitioned into adult services.

To compare our practice with national and international guidelines. $^{1,2,3,4,5}$

To undertake cost analysis for various screening strategies.

Methods A complete list of all known T1D children and young persons within our Trust in 2017 was obtained. The upper age limit was restricted to 18 years. A pre-designed proforma was used to record demographic information including sex, current age, age at diagnosis and current coeliac status. We also recorded how often and what method was used to undertake coeliac surveillance. This was compared with national and international recommendations for CD in highrisk groups.

Simultaneously we sent out a questionnaire to all other children and young people's services in the region regarding their screening and surveillance practices for CD in T1D children and young people. The results obtained were compared with our practice.

We undertook economic evaluation for various screening strategies 